\title{
L'entrepreneuriat collectif au service des communautés dévitalisées de la Mauricie : un modèle inspirant
}

\author{
Paul Prévost, Ph. D. \\ Université de Sherbrooke \\ René Bougie, Master 1 Science Po et Sociales et M. Adm. En gestion et gouvernance des \\ coopératives et des mutuelles
}

\section{INTRODUCTION}

\section{En raison des caractéristiques intrinsèques des sept municipalités, il a été pris pour hypothèse que ces communautés nécessitent un accompagnement adapté pour renforcer leur capacité à se développer. Le projet pilote a donc servi à doter ces municipalités d'une ressource qui peut pallier à ce manque d'expertise, de connaissances et de ressources techniques, s'il y a lieu, dans le développement de projets d'entreprises collectives.}

«Le développement de l'emploi par l'entrepreneuriat collectif dans les milieux dévitalisés » est un projet original qui a été initié par EmploiQuébec en 2009, en collaboration avec la Coopérative de développement régional Centredu-Québec/Mauricie (CDRCQM), le ministère du Développement économique, de l'Innovation et de l'Exportation (MDEIE), le ministère des Affaires Municipales, des Régions et de l'Occupation des Territoires (MAMROT), et la Conférence Régionale des Élus de la Mauricie (CRÉ). D'une durée de trois ans, il visait le support à la revitalisation de sept municipalités de la région de la Mauricie considérées très dévitalisées selon des données des recensements 2001 et 2006 et une analyse faite par le MAMROT.

Ces sept communautés sont Lac Édouard, NotreDame-de-Montauban, Saint-Alexis-des-Monts, StÉdouard-de-Maskinongé, Ste-Thècle, Ste-Angèlede-Prémont et Trois-Rives. Le processus de revitalisation s'est effectué par le biais de la création d'emplois au sein de nouvelles entreprises collectives.
En raison des caractéristiques intrinsèques des sept municipalités, il a été pris pour hypothèse que ces communautés nécessitent un accompagnement adapté pour renforcer leur capacité à se développer. Le projet pilote a donc servi à doter ces municipalités d'une ressource qui peut pallier à ce manque d'expertise, de connaissances et de ressources techniques, s'il y a lieu, dans le développement de projets d'entreprises collectives.

L'encadrement du projet pilote s'est effectué au quotidien par la Coopérative de développement régional Centre-du-Québec/Mauricie (CDRCQM) qui s'est appuyé sur un comité technique et un comité aviseur formés par des membres des organismes parrains du projet pilote. Cette équipe composant le dispositif d'intervention du projet pilote s'est distinguée particulièrement par le rôle proactif de son comité aviseur, le rôle très actif des membres du comité technique et des comités de développement local et enfin par le recrutement d'un conseiller en entrepreneuriat collectif capable d'œuvrer en amont (et en synergie) des agences de développement local régulières pour conscientiser les populations locales et faire émerger des projets collectifs.

La combinaison de ces éléments a permis l'émergence d'un véritable dispositif permettant un support et des ramifications uniques, tout en offrant une grande souplesse au niveau de l'action du conseiller en entrepreneuriat collectif dans les communautés retenues dans le projet pilote. Une équipe de recherche a été impliquée tout au long du projet. 


\section{PEUT-ON TIRER DES LEÇONS D'UNE EXPÉRIENCE UNIQUE?}

Il est toujours tentant de répliquer d'une région à une autre un modèle qui a eu du succès. N'est-ce pas là aussi le but des expériences pilotes? Cela peut toutefois être dangereux, car on ne pourra pas répliquer facilement les personnes qui ont fait la différence en Mauricie, ni leur complicité, ni leur motivation. De plus, bien que les communautés dévitalisées réunissent un certain nombre de caractéristiques communes sur le plan des indicateurs de développement, du besoin d'accompagnement, de transfert des savoirs, du réseautage, et de la culture entrepreneuriale, on ne peut pour autant prendre pour hypothèse qu'elles sont totalement sembla-

\section{LA DÉMARCHE}

Le projet pilote a suivi une démarche qui, bien qu'adapté à ses objectifs, ne se démarque pas particulièrement des stratégies et pratiques reconnues en développement local, développement social, développement économique communautaire ou économique et social et en développement des communautés. À la base, toutes ces stratégies reposent sur la reconnaissance que les collectivités sont capables de prendre en charge leur développement. Toutefois, sa mise en application dans le projet pilote a permis de mettre en relief toute une variété d'apprentissages et de leçons susceptibles d'inspirer d'autres expériences centrées sur le développement de l'entrepreneuriat collectif.

\subsection{La mise en situation}

La sélection des collectivités participantes s'est effectuée sur la base d'indicateurs développés par le MAMROT. Il est vite apparu qu'il fallait enrichir cette information si on voulait bien comprendre les problématiques particulières de chaque municipalité, leurs capacités et les dynamiques qui les animent. Car, en fin de compte, pour initier un processus de revitalisation durable, il a été rapidement réalisé qu'il fallait plus que tenter de créer de nouvelles structures ou de nouvelles entreprises, il fallait aussi essayer d'influencer le comportement des acteurs, en développant de nouvelles capacités et de nouvelles valeurs telles, la solidarité, la coopération, l'équité, la démocratie, etc. À l'occasion, on a même considéré le projet pilote comme une sorte de projet d'éducation des adultes, d'éducation bles. Ce n'était pas le cas en Mauricie, ce ne le sera pas plus ailleurs.

Cette mise en garde effectuée, il y a bien tout de même quelques leçons à tirer. En essayant de minimiser les particularités liées au contexte et aux personnalités, voici une tentative de post rationalisation sur les deux plus importantes dimensions structurant l'expérience vécue en Mauricie au cours des trois dernières années soient la démarche qui a été opérationnalisée et le dispositif d'intervention mis en place.

populaire. Ces informations plus qualitatives qui ont été assemblées lors de la première année du projet pilote ont permis entre autres de comprendre pourquoi certaines municipalités nécessitaient plus de temps que d'autres pour démarrer ou avancer plus lentement. La dévitalisation plus récente par exemple n'a pas le même poids qu'une dévitalisation enracinée depuis plus longtemps ni le même impact sur la démobilisation de la collectivité. L'intervention doit se situer sur la trajectoire historique des municipalités et miser sur le vécu du territoire pour imaginer l'avenir. Cela devrait conditionner les stratégies d'intervention et d'accompagnement, le calendrier de réalisation ainsi que l'évaluation des processus et des résultats.

\subsection{Le contact avec les élus et la municipalité}

Le projet-pilote pourrait être qualifié d'exoendogène, c'est-à-dire provoqué de l'extérieur de la collectivité, mais reposant en définitive sur des initiatives issues des populations locales. Donc le lancement du projet-pilote a dû être négocié avec des instances locales. Si certaines collectivités ont sauté d'emblée sur l'opportunité, d'autres ont offert un accueil beaucoup plus froid, voire même un refus. Comment expliquer ces fermetures pour créer des conditions favorables à l'ouverture?

Les petites municipalités et les municipalités dévitalisées possèdent généralement peu d'institutions et d'associations. Donc si on veut intervenir sur leur territoire, on est rapidement en contact avec la municipalité, les élus locaux et la direction générale. Il n'y a virtuellement pas de détour 
possible, ils ont quelques ressources et représentent un leadership certain. La collaboration avec des élus est souvent délicate, car s'ils veulent le bienêtre de leur collectivité, ils sont aussi soumis à un agenda de quatre ans et à un processus électoral. Donc en gros, à la première année du mandat, l'énergie n'est pas encore complètement focalisée, la deuxième et la troisième sont les années potentiellement les plus productives, alors que la quatrième sera graduellement distraite par les élections qui approchent. Il faut composer avec cette réalité. Ceci dit, chaque élu a aussi sa personnalité, sa vision du développement (vision économique ou sociale, conservatrice ou innovante, individuelle ou collective), ses compétences et ses intérêts. Certains sauront saisir l'opportunité que représente un projet comme le projet pilote, d'autres y verront une distraction et enfin, certains le considéreront comme une menace susceptible d'alimenter une éventuelle opposition. Si dans le dernier groupe peu d'entre eux s'opposeront directement au projet, ils sauront par contre diluer leur collaboration de mille manières.

Évidemment, collaborer avec des élus visionnaires, impliqués et bien intentionnés, c'est l'idéal. Pour se rapprocher des élus et obtenir leur appui, il faut parfois s'adresser à leur réseau de confiance, des personnes crédibles à leurs yeux. L'une d'elles est certainement la direction de la municipalité, un poste clé dans toutes les municipalités.

Évidemment, collaborer avec des élus visionnaires, impliqués et bien intentionnés, c'est l'idéal. Pour se rapprocher des élus et obtenir leur appui, il faut parfois s'adresser à leur réseau de confiance, des personnes crédibles à leurs yeux. L'une d'elles est certainement la direction de la municipalité, un poste clé dans toutes les municipalités. Ces personnes pourront vraiment faciliter le contact avec les élus concernés ainsi que leur collaboration. Dans le projet pilote, à cet égard, on a fait appel à des membres des comités aviseur et technique pour expliquer et lancer le projet dans chaque municipalité, puis à l'occasion, ils sont revenus pour prêter main-forte pour clarifier les orientations du gouvernement, insister sur la contribution du milieu, etc. Dans d'autres cas, l'aide est venue de certains partenaires du développement.
Rappelons-nous que les élus dans les petites collectivités travaillent presque de façon bénévole et disposent de ressources limitées pour remplir leur mandat. De plus, comme leur participation et leur engagement sont presque toujours essentiels au développement des collectivités locales, une bonne connaissance de la vie politique locale est donc toujours un atout.

\subsection{La formation d'un comité de dévelop- pement local et la mobilisation citoyenne}

Dans une démarche de revitalisation, la participation et le bénévolat jouent un rôle important, mais ils s'épuiseront s'ils ne sont pas supportés par un minimum d'organisation. Rapidement, dans le projet pilote, les municipalités participantes se sont dotées, à la faveur d'une consultation publique, d'un comité de développement local. Ce comité voulait réunir des acteurs de la collectivité qui avait le goût de s'impliquer et qui avait des idées de projet. Il voulait aussi assurer une sorte de permanence et faciliter la rotation des bénévoles. On y est arrivé avec des succès variables. LacÉdouard a même transformé son comité de développement local en une coopérative de développement. Dans d'autres cas, il a été difficile de l'animer et de maintenir la participation. Idéalement, ce comité devrait être représentatif des forces vives de la collectivité et regrouper des élus, des leaders, des représentants de la classe affaires et de la société civile ainsi que des citoyens. Assez nombreux pour représenter une force d'action, il doit être assez petit pour être efficace et maintenir l'implication de tous. Ce n'est pas un groupe politique ni un groupe de pression, c'est un comité qui devrait carburer à la créativité et à l'action. Toutes des vertus qui ne sont pas faciles à pratiquer sur le terrain. La communication à l'intérieur du comité et avec la collectivité de même que la transparence, le tout couplé au doigté du conseiller en entrepreneuriat collectif, sont des ingrédients clés du succès à ce niveau.

Malgré tout, ce n'est pas un exercice évident, car pour y arriver il faudra conjuguer des rationalités d'affaires avec des rationalités politiques ainsi qu'avec des rationalités associatives et communautaires pour partager ensemble une même culture entrepreneuriale et le même goût pour le développement de sa communauté. 


\section{4 Échanges et mimétisme}

Lors du deuxième atelier de transfert, le travail s'est centré sur des thématiques plus pointues jugées particulièrement prenantes par les participants soient : l'organisation et la gestion de projet, la participation et la mobilisation citoyenne, l'accompagnement, le support et le réseautage ainsi que le financement des entreprises collectives.

Les échanges entre les participants, le conseiller en entrepreneuriat collectif et les autres partenaires du projet pilote ont permis des apprentissages tout au long de l'expérience. Mais, malgré l'empathie du conseiller en entrepreneuriat collectif, la relation établie en est une d'accompagnant à accompagnés. Pour enrichir l'exercice, les participants ont été amenés à échanger avec des acteurs participant à d'autres expériences québécoises de développement local et de développement coopératif. C'est ainsi que plusieurs se sont déplacés à StCamille et à la Coopérative de St-Ubalde par exemple pour partager de visu avec des acteurs terrains comme eux. Les apprentissages retirés sont différents parce que les échanges se font entre «collègues » d'égal à égal et stimulent l'effet de mimétisme.

Toujours dans cette perspective, dans le projet pilote, deux ateliers de transfert ont été tenus à l'an 2 et 3 du projet. L'objectif de ces ateliers était de favoriser les échanges entre les acteurs des collectivités participantes, de stimuler les troupes en partageant les bons résultats comme les difficultés et de favoriser le réseautage. Lors du premier atelier, chaque collectivité participante a présenté en plénière ses initiatives et l'état de l'avancement des travaux en cours puis, en petit groupe, l'attention s'est déplacée vers le partage des questions, difficultés et bons coups de chacun. $\mathrm{Ce}$ contact plus direct a permis par exemple d'identifier des faiblesses communes au niveau de la communication entre le CDL et la collectivité et la nécessité d'accentuer le rôle des élus. Ce qui a donné lieu entre autres au recrutement d'une spécialiste en communication qui est venue donner un coup de main aux CDL intéressés. Lors du deuxième atelier de transfert, le travail s'est centré sur des thématiques plus pointues jugées particulièrement prenantes par les participants soient: l'organisation et la gestion de projet, la participation et la mobilisation citoyenne, l'accompagnement, le support et le réseautage ainsi que le financement des entreprises collectives. En petits groupes puis en plénière, les participants, plus avancés dans leurs différents projets que lors de l'atelier précédent, ont discuté de leurs pratiques, des défis et des difficultés à mener des projets concrets, à construire des montages financiers, à obtenir la participation citoyenne, la collaboration des élus, à effectuer des campagnes de souscription populaire pour aider au financement, etc. On a aussi abondamment discuté de pérennité. Qu'est-ce qui arrive après le projet pilote? Comment construire sur cette lancée?

Ces efforts d'échange et de partage sont apparus comme des exercices d'apprentissage jugés très profitables de l'avis des participants. Ils ont même suscité une solidarité dont les effets se sont fait sentir jusqu'aux instances régionales.

\subsection{Des projets d'entreprises de services de proximité}

Dans le cadre du projet pilote, plusieurs projets d'entreprises se sont adressés à des services de proximité et se sont organisés en coopérative de solidarité. Est-ce strictement contextuel ou y a-t-il là quelque chose de plus significatif ou de plus intéressant?

D'emblée, on peut convenir en accord avec la littérature en économie que normalement une entreprise de service de proximité ne joue pas de rôle moteur et a peu ou pas d'effets multiplicateurs dans une collectivité. Toutefois, dans le cas d'une communauté dévitalisée qui a perdu ou est en voie de perdre la majorité de ses services de proximité, ces enjeux deviennent stratégiques. Comment maintenir sa population, attirer entreprises et travailleurs sans l'existence d'un milieu de vie suffisamment attrayant, suffisamment organisé? Ce choix prend alors une tout autre signification pour une collectivité qui décide de donner un coup de barre et de relancer son développement. De plus, au début d'un processus qui s'enclenche, il est plus facile de mobiliser la population sur des besoins collectifs et de proximité, car tout le monde se sent concerné dans son quotidien. Des résultats positifs seront susceptibles d'améliorer le 
sentiment d'appartenance et le goût d'entreprendre d'autres projets de plus en plus structurants tant sur le plan économique que social. L'exemple de la création de la coopérative de solidarité de NotreDame-de-Montauban est éloquent à cet égard. S'il faut sonner le réveil de la communauté, cela semble moins risqué tout en étant une bonne école de formation à l'entrepreneuriat.

$\mathrm{Si}$ le projet pilote devait favoriser les projets d'entrepreneuriat collectif, aucun mode d'organisation particulier n'a été privilégié à priori. Toutefois, la formule des coopératives de solidarité s'est imposée comme un mode d'organisation de choix par les collectivités incluses dans le projet pilote. La coopérative de solidarité permet un partenariat élargi, impose un «membership», des rapports annuels publics et facilite les premières mises de fonds collectives, ce qui s'est avéré un atout majeur pour obtenir l'appui des bailleurs de fonds et des municipalités concernées. À titre d'exemple, les coopératives multiservices de Trois-Rives, de Lac Édouard et de Notre-Dame-de-Montauban ont recueilli des sommes significatives auprès de leurs membres.

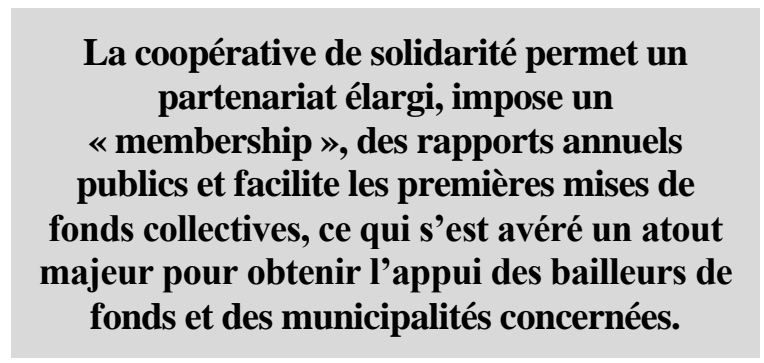

\subsection{Le financement des projets}

Le financement des projets a constitué l'un des facteurs de stress les plus élevés chez les promoteurs de projet. En effet, la très grande majorité des citoyens ne sont pas au fait des programmes existants et des modalités les encadrant et les agents de développement qui les accompagnent ne sont pas tous des spécialistes en la matière. Ces derniers, en travaillant sur divers projets, s'échangent des informations entre eux sur les possibilités de financements disponibles et sur les règles et les modalités qui encadrent leur accès. Une chose est sûre, les divers acteurs ne doivent pas hésiter à faire appel aux personnes ressources qui sont spécialisées dans le domaine sur leur territoire.
Dans l'actuel projet pilote, les communautés ayant développé un projet nécessitant un financement avaient l'obligation de contribuer à la hauteur de 20 à $25 \%$ en parts provenant de la population locale dans le montage financier de leur initiative. Étant dans de petites communautés, amasser des sommes oscillant entre $35000 \$$ et $130000 \$$ n'a pas été une mince affaire. Pour ce faire, afin de faire face à cette difficulté particulière, les divers promoteurs ont illustré l'utilité de ne pas hésiter à faire preuve de créativité et d'innovation pour réussir à recueillir les sommes nécessaires à la participation des bailleurs de fonds.

\subsection{D'une approche projet à une vision stratégique}

$\mathrm{Si}$, normalement, un effort de développement particulier doit s'initier autour d'une mise en situation et d'un exercice de planification, cela n'a pas été le cas dans la majorité des municipalités participantes (certaines avaient toutefois déjà un plan stratégique). Dans les faits, rapidement, on est parti en quête de projets pour passer à l'action sans délai. Dans le contexte, cette stratégie était pleine d'allure, car les projets concrets apparaissaient plus mobilisateurs pour une population peu rompue ou peu intéressée aux grandes réflexions sur le développement. L'hypothèse étant qu'après les premiers succès, les collectivités participantes voudraient naturellement faire le point pour donner un sens plus large à leurs actions et identifier de nouvelles initiatives conséquentes (ce fut le cas en particulier de Lac Édouard). Le risque toutefois, c'est que le comité de développement local se transforme en comité de projet, concentre toutes ses énergies uniquement sur le projet en cours et considère son mandat terminé après sa réalisation. Auquel cas, il faudra recommencer le travail d'animation au tout début.

Pour éviter ce cul-de-sac, il faut au moment opportun inscrire le travail de chaque communauté dans une perspective à moyen et long terme en facilitant le développement d'une vision, d'un plan stratégique et d'un portefeuille de projet, des petits et des plus gros de façon à développer non seulement le milieu de vie, ce qui est plus facile au début, mais aussi à faciliter la reconversion économique de la communauté. 
2.8 La communication avec les partenaires et les citoyens

La communication revêt une importance primordiale au sein des communautés dévitalisées, tout particulièrement lorsqu'il est question de mettre en ouvre des projets de nature collective.

La communication revêt une importance primordiale au sein des communautés dévitalisées, tout particulièrement lorsqu'il est question de mettre en œuvre des projets de nature collective. Cet aspect de la gestion de projet, identifié par les acteurs euxmêmes lors du premier atelier de transfert comme étant un facteur de réussite quasi incontournable, s'est révélé être un défi pour plusieurs communautés.

En plus du rôle essentiel de la communication dans la mobilisation citoyenne, nous avons noté que les communautés qui utilisaient leur média local (Notre-Dame-de-Montauban, St-Alexis) pour diffuser leurs messages ont réussi à éviter dans une plus grande mesure les imbroglios et les impacts négatifs liés à la pratique du bouche-à-oreille ou du téléphone arabe.

De plus, l'adoption d'un plan de communication formelle par un CDL (St-Alexis-des-Monts) a redynamisé ses membres et leur a permis de recentrer leurs actions. En somme, il peut s'avérer intéressant, voire indispensable de susciter l'intérêt de la population envers un projet collectif en utilisant diverses formes de communication (séance d'information, article dans le journal local, présence à des activités sociales) à travers un canal de communication clair et transparent porté par une ou des personnes crédibles, légitimes et convaincantes.

\subsection{Le développement ça prend du temps}

L'expérience pilote démontre que le temps investi pour identifier un projet d'entreprise collective basé sur les besoins du milieu a nécessité de huit à dix mois de concertation (idées individuelles versus vision collective, leadership, assemblée citoyenne, campagne de recrutement de membres ou campagne de souscription, stratégies de communication, etc.). Le processus de démarrage d'une entreprise collective pour sa part représente environ une à deux années de travail concerté (connaissance du milieu, mobilisation, formation d'un comité, constitution d'une entreprise collective (Coop ou OBNL), élaboration d'un plan d'affaires, élaboration d'un plan d'action, stratégies de communication, réalisation de projet, gestion financière, etc.). Le processus collectif apparaît très long comparativement au modèle traditionnel de l'entreprise privée. Il faut retenir qu'au sein des petites communautés, les personnes engagées sont souvent les mêmes. De plus, même si l'expérience pilote a impliqué dans ces démarches autant des élus, des citoyens, des personnes et des organisations, chacun a modulé sa participation selon ses capacités, ses connaissances et ses compétences et tous ont fonctionné en mode apprentissage à plusieurs égards.

Si la mise sur pied des processus visant à voir émerger divers projets en entrepreneuriat collectif ont permis, dans une certaine mesure, d'influencer positivement l'acquisition de nouvelles capacités de changement et d'intervention dans les diverses communautés visées par le projet pilote, elle n'a pas encore permis de l'avis de tous de développer l'autonomie et la résilience essentielles au développement local. Cela prend plus de temps et de patience pour terminer la phase de décristallisation et transiter vers un nouvel équilibre ou chaque communauté revitalisée aura les capacités pour participer activement à son propre développement aussi bien sur le plan social, culturel qu'économique. Trois ans d'effort concerté apparaissent un peu courts malgré les bons résultats pour baisser les bras ou même réduire les efforts.

\footnotetext{
Si la mise sur pied des processus visant à voir émerger divers projets en entrepreneuriat collectif a permis, dans une certaine mesure, d'influencer positivement l'acquisition de nouvelles capacités de changement et d'intervention dans les diverses communautés visées par le projet pilote, elle n'a pas encore permis de l'avis de tous de développer l'autonomie et la résilience essentielles au développement local.
} 


\subsection{La gestion de projet sur le terrain}

Avoir des idées innovatrices c'est quelque chose, mais les transformer en projet concret reste un défi de taille. Avec l'aide du projet-pilote, toutes les communautés participantes se sont lancées dans l'aventure. Elles ont appris à la dure et, à leur avis, si c'était à recommencer, elles feraient autrement et s'inscriraient entre autres dans des démarches plus systématiques ou feraient appel plus tôt à de l'expertise ou à des collaborations externes. Par exemple à Notre-Dame-de-Montauban, pour développer leur coopérative multiservice, les promoteurs ont recruté un directeur de chantier compétent et se sont associés à la Coopérative de consommation de Saint-Ubalde. Le renforcement des compétences au niveau de l'organisation et la gestion de projet ainsi que la construction d'un coffre d'outils qui retiendrait ces apprentissages et d'autres pourrait faciliter la tâche des promoteurs locaux à l'avenir.

\subsection{Réflexivité et recherche}

Animer une démarche de développement local n'est pas un processus linéaire, c'est une expérience humaine complexe, ou les rebondissements

\section{LE DISPOSITIF D'INTERVENTION}

La grande originalité du projet pilote mauricien est le dispositif d'intervention mis en place pour tenter de venir en aide à sept communautés dévitalisées de la région. Ainsi, le projet a été coordonné par la CDR et chapeauté par deux comités soient un comité aviseur et un comité technique. Le conseiller en entrepreneuriat collectif, la cheville ouvrière du projet a complété le dispositif. Au-delà des personnes et du contexte, peut-on tenter de dégager des éléments sinon transférables du moins inspirants $\mathrm{du}$ travail de ce dispositif d'intervention? Quatre éléments nous sont apparus particulièrement structurants : un parrainage efficace, un conseiller en entrepreneuriat collectif au mandat souple, une cible d'intervention bien ajustée aux compétences des partenaires locaux et régionaux et un réseautage permettant une grande synergie et une grande résilience.

\subsection{Un parrainage efficace}

Le comité aviseur, le comité technique et la CDR qui chapeautaient l'expérience pilote ont offert en sont nombreux et les ajustements sont presque quotidiens. Si on peut s'inspirer d'expériences similaires et se documenter dans la littérature sur le sujet pour se développer des stratégies et articuler ses pratiques, il y aura toujours, dans chaque collectivité, une variété d'aspects uniques au niveau du contexte et des acteurs en présence. Il est donc essentiel de se donner des moments de réflexion pour jeter un regard critique sur ses orientations, ses pratiques, ses relations et interrelations, sur ses compétences, bref sur tout son modèle de développement.

Dans le projet pilote, cet exercice s'est fait de façon continue dans le rapport particulier entretenu entre l'équipe de recherche, le conseiller en entrepreneuriat collectif et le comité technique. Il s'est poursuivi au niveau des CDL de façon spéciale lors des ateliers de transfert. C'est une illustration, d'autres façons de faire pourraient évidemment convenir, l'important c'est de reconnaitre la fluidité et la souplesse du développement local et la nécessité conséquente de questionner régulièrement ses stratégies et ses pratiques et, d'apprendre.

permanence, en plus d'un appui moral, un accès privilégié à une expertise stratégique et à une expertise technique crédibles et reconnues chez les élus et les leaders des collectivités participantes ainsi que chez les partenaires impliqués. Ils ont aussi donné d'emblée accès à leurs réseaux de contacts respectifs et enfin, ils n'ont pas hésité (comité technique et CDR en particulier) à retrousser leurs manches pour se déplacer sur le terrain et appuyer le conseiller en entrepreneuriat collectif quand cela a été jugé nécessaire. Enfin, ils ont su prendre des risques politiques et financiers pour innover autant au niveau de leur structure que sur le terrain. Ce parrainage peut prendre d'autres formes dans d'autres régions, mais il ne peut avoir moins d'implication ni réunir au total moins de compétence et de crédibilité.

\subsection{Un conseiller en entrepreneuriat collectif au mandat souple}

Le conseiller en entrepreneuriat collectif a formé la pierre angulaire du dispositif d'intervention mis en 
place en Mauricie. Relié à aucun programme précis, il avait un mandat souple qu'il pouvait moduler au gré des problématiques particulières, des interventions et des partenariats suscités. À cette liberté d'action, s'ajoutent une variété d'habilités telles l'empathie, la détermination et la résilience. Il faut ajouter aussi une vision stratégique sociale et économique de sa tâche, la capacité de se mouvoir entre les inévitables conflits qui surgissent sur le terrain et la capacité de soutenir la motivation et de maintenir le cap vers la réalisation de résultats concrets. N'oublions pas aussi la capacité de travailler en équipe avec des élus, des leaders, des gens d'affaires, la société civile et les autres agents de développement sur le territoire, bref, la capacité de mobiliser les ressources et de susciter les partenariats nécessaires à l'émergence de stratégies et de projets de développement significatifs. Enfin, son travail sera inévitablement handicapé s'il n'a pas aussi le profond souci de maintenir une grande complicité avec son comité de parrainage. Il a agi donc plus comme un généraliste que comme un spécialiste du développement des collectivités, de là l'importance d'avoir accès à un réseau de support varié et efficace.

\section{Le conseiller en entrepreneuriat collectif a formé la pierre angulaire du dispositif d'intervention mis en place en Mauricie. Relié à aucun programme précis, il avait un mandat souple qu'il pouvait moduler au gré des problématiques particulières, des interventions et des partenariats suscités.}

\subsection{Une cible d'intervention bien ajustée}

Toutes les régions et MRC du Québec possèdent une variété d'agence de développement et d'intervenants sur leur territoire. Il devient important d'éviter de dupliquer les efforts et de créer un environnement compétitif aux effets nocifs. Dans le projet pilote de la Mauricie, ce souci a été omniprésent. Il est toutefois rapidement apparu que les petites collectivités et les collectivités dévitalisées n'ont pas toujours les connaissances ni les ressources pour faire émerger des projets et obtenir un support conséquent. C'est ainsi que les interventions du conseiller en entrepreneuriat collectif $\mathrm{du}$ projet pilote se sont concentrées en amont, se sont effectuées en complémentarité avec le travail des agents de développement déjà sur le territoire et, ont construit sur une mise en relation de l'implication citoyenne et de l'implication municipale. Un endroit qui concentre l'attention sur le développement des capacités de la collectivité avant et en appui au développement des projets. Un moment qui demande plus de temps et beaucoup de souplesse de la part des intervenants. Évidemment, la fixation des objectifs et l'évaluation des résultats d'une telle intervention devront tenir compte de cette situation particulière. Les bailleurs de fonds aiment habituellement concentrer leur attention sur des indicateurs quantitatifs tels le nombre d'emplois créés, le nombre d'entreprises lancées ou la quantité de dollars investis; bref, des indicateurs à court terme. Dans ce cas-ci, c'est insuffisant pour évaluer les résultats des efforts consentis. Il faut ajouter d'autres indicateurs plus qualitatifs capables d'évaluer des processus, l'accroissement des capacités de développement de la collectivité, l'émergence de nouvelles dynamiques et de nouvelles valeurs. Sinon le travail d'animation, d'éducation et de renforcement du conseiller en entrepreneuriat collectif se réduira comme une peau de chagrin sous les pressions pour une atteinte de résultats quantitatifs à tout prix.

\subsection{Réseautage et synergie}

Le travail du conseiller en entrepreneuriat collectif est exigeant et personne ne peut posséder toutes les qualités et toute l'expertise requises pour une tâche aussi délicate et aussi variée. Il faut donc que le conseiller en entrepreneuriat collectif soit dans un temps respectable, capable de les réunir en faisant appel à son réseau et aux réseaux de ses alliés, le comité de parrainage et les partenaires régionaux et locaux du développement. C'est la synergie issue de cette collaboration qui pourra assurer l'efficacité du travail du conseiller en entrepreneuriat collectif et appuyer de façon durable les efforts de développement qui seront consentis dans les collectivités impliquées. Dans le projet pilote, le conseiller en entrepreneuriat collectif a changé trois fois, le directeur de la CDR, l'assise institutionnelle de celle-ci a aussi changé trois fois et deux membres très actifs du comité technique ont pris une année sabbatique lors de la troisième 
année du projet. Chacun de ces changements aurait dû tuer le projet. Il a tout de même survécu pour se transformer en succès parce qu'il a été plus que le résultat des efforts d'une seule personne, soit du conseiller en entrepreneuriat collectif comme il l'avait été anticipé au début. C'est aussi le résultat de la synergie créée dans tout le dispositif d'intervention (comité aviseur, comité technique,

\section{CONCLUSION}

Toutes les municipalités ont travaillé avec leurs atouts disponibles et ont en général amélioré sensiblement leurs capacités ou potentiels d'intervention et de changement. De plus, plusieurs ont accru de façon considérable leur capacité à se réseauter, à faire appel aux ressources de l'État et, dans une certaine mesure, à augmenter leur capacité de résilience face à l'adversité.

Le but d'un projet-pilote est d'explorer de nouvelles avenues et de tirer des leçons. Dans le projet de la Mauricie, à la faveur de divers constats, on avait pris pour hypothèse qu'en dotant les municipalités dévitalisées de la région du support d'un agent de développement en entrepreneuriat collectif au mandat très souple on pourrait contribuer à les relancer sur le chemin de la revitalisation.

Lorsque l'on porte un regard d'ensemble sur le projet ayant eu cours en Mauricie, on peut affirmer sans risque de se tromper que, du point de vue des objectifs quantitatifs fixés par le projet, le projet a rencontré la majorité des attentes fixées en la matière. En effet, bien que ce ne soit pas toutes les municipalités qui se soient dotées d'une entreprise collective, l'objectif en matière d'employabilité a, après trois ans et trois mois, grandement été surpassé en atteignant 25 emplois directs créés et maintenus.

Une analyse des objectifs qualitatifs établit au cours du projet illustre également que l'idée d'implanter un dispositif d'intervention original dans le but de développer des entreprises collectives visant à répondre aux besoins des communautés locales a été excellente. Bien que chaque communauté n'ait pas eu une situation de départ similaire et, ce faisant, n'ait pas disposé du même capital stratégique, chacune a su tirer profit de cette expérience. Toutes les municipalités ont
CDR et le conseiller en entrepreneuriat collectif) et la collaboration obtenue des comités locaux de développement et des partenaires qui ont permis de passer à travers ces difficultés et d'atteindre les résultats espérés. Notons que les comités locaux de développement n'ont tout de même pas été laissés trop longtemps sans support durant ces turbulences.

travaillé avec leurs atouts disponibles et ont en général amélioré sensiblement leurs capacités ou potentiels d'intervention et de changement. De plus, plusieurs ont accru de façon considérable leur capacité à se réseauter, à faire appel aux ressources de l'État et, dans une certaine mesure, à augmenter leur capacité de résilience face à l'adversité.

Toutefois, si l'agent de développement en entrepreneuriat collectif a joué un rôle significatif, il n'aurait pu le faire sans le positionnement judicieux de son champ d'action et l'établissement d'une collaboration constante avec les acteurs du milieu, les comités de parrainage, en particulier le comité technique, et les partenaires régionaux et locaux du développement

Enfin, bien que ce projet ait constitué un succès en soi, il peut difficilement être considéré comme une expérience achevée de revitalisation de communautés. On peut en effet douter que les communautés participantes aient toutes acquis à la fin du projet pilote suffisamment d'autonomie pour assumer elles-mêmes dans le futur le pilotage complet de leur projet de revitalisation. Le développement n'est pas une mécanique qu'on peut actionner à volonté, c'est un processus de transformation profond qui touche la culture et les capacités d'une collectivité. Selon le niveau de dévitalisation, cela peut prendre plus de trois ans d'accompagnement soutenu sinon ce sera un coup d'épée dans l'eau. On ne peut tout simplement pas espérer qu'un projet d'accompagnement trop court s'attaque efficacement aux difficultés sousjacentes à la dévitalisation et règle les problèmes auxquels doivent faire face les communautés touchées par le projet-pilote. En résumé, à notre avis, ce projet est un pas dans la bonne direction, mais, pour arriver à la fin du parcours, les acteurs ne devraient pas cesser leur marche. 


\section{BIBLIOGRAPHIE}

Alvord.S.H., Brown.L.D. et Letts.C.W., (2004). «Social entrepreneurship and societal transformation: An exploratory study ", The Journal of Applied Behavioral Science, Vol. 40, N 3, pp.260-283.

Aradel, Créer et animer des réseaux : entre savoir être et savoir faire des développeurs économiques, 6 (2004), 58 pages.

Beckley M., T., Martz, D., Nadeau, S., Wall, E., Reimer, B., Multiple Capacities, Multiple Outcomes: Delving Deeper into the Meaning of Community Capacity, Journal of Rural and Community Development, 3 (2008), 56-75.

Boisvert, R. (2007). Les indicateurs de développement des communautés : Vers le déploiement d'un dispositif national de surveillance. Cahier technique présenté aux participants de l'atelier méthodologique des JASP 2007.

Chantier de l'économie sociale, Mémoire présenté dans le cadre de la tournée de consultation sur la Stratégie québécoise de l'entrepreneuriat «Vers une stratégie de l'entrepreneuriat » au MDEIE, 2010, 12 pages.

Chantier de l'économie sociale, Outil de sensibilisation à l'économie sociale, Une économie à valeurs ajoutées, 2004, 28 pages.

Dumais, L., Camus, A., et Tremblay, J-M., «L'évolution de la pratique des agents d'économie sociale à Montréal » - Rapport de recherche réalisé dans le cadre des activités du Groupe de recherche et d'activités partenariales de Montréal et du Réseau québécois de recherche partenariale en économie sociale, Cahier No RQ-07 (2011), 59 pages.

Forest, D., St-Germain, L. (2010), La participation citoyenne, le point de vue de citoyennes et de citoyens $\mathrm{du}$ nord de Lanaudière sur leur participation dans les démarches d'animation territoriale en développement durable, CSSSNL, 124 pages.

Greffe, X. (2002). Le développement local. La Tour d'Aigues (France) : Éditions de l'Aube.

Groupe de travail sur les communautés dévitalisées, Des communautés à revitaliser. Un défi collectif pour le Québec, 54 pages.

Martin, A., Molina, E, Lafleur, M., le paradigme coopératif : proposition renouvelée pour répondre aux attentes de la société actuelle, Cahier de recherche de l'IRECUS, 1 (2008), 21 pages.

Omrane, A., L'entrepreneuriat social et le développement durable : quels modèles d'affaires dans le champ social?, (en ligne), http://www.strategieaims.com/events/conferences/2-xixeme-conference-de1-aims/communications/85-lentrepreneuriat-social-et-ledeveloppement-durable-quels-modeles-daffaires-dansle-champ-social/download.
Prévost, P. (1993). Entrepreneurship et développement local : quand la population se prend en main. Montréal : Les Éditions Transcontinentales inc.

Prévost, P. (2001). Le développement local : Contexte et définition. Sherbrooke : IRECUS.

Reimer, B., The rural context of community development in Canada, Journal of Rural and Community Development, 1(2006) 155-175.

Solidarité rurale du Québec (2003). Les savoirs collectifs locaux. Revue d'initiatives locales de développement en milieu rural, Nicolet. SRQ.

Thompson J. (2002). "The world of the social entrepreneur», The International Journal of Public Sector Management, Vol. 15, No 4-5, pp. 412-431.

Yorn, C., Lussier, K. et Prévost, P. (2007). Bâtir ensemble notre région - rapport intégral. Chibougamau. Centre régional de santé et de services sociaux de la Baie-James, Direction de santé publique.

Yorn, C., Lussier, K. et Prévost, P. (2008). Le capital stratégique des communautés jamésiennes.

Organisations et Territoires, 17(01), 53-60.

\section{Références}

Prévost Paul, Veillette Lucie. Rapport de mise en situation : Analyse transversale présenté à la Coopérative de développement régional Centre-du-Québec/Mauricie dans le cadre du projet Développement de l'emploi par l'entrepreneuriat collectif. 76 p. Sherbrooke, janvier 2011.

Bougie René, Prévost Paul. Rapport final, présenté à la Coopérative de développement régional Centre-duQuébec/Mauricie dans le cadre du projet Développement de l'emploi par l'entrepreneuriat collectif dans les municipalités dévitalisées de la Mauricie, 75 p. Sherbrooke, avril 2012. 